\title{
MICROBIOLOGY
}

\section{More than microbial relative abundances}

The concept of reference frames inspires researchers to develop a differential ranking system for measuring relative differential abundance, which does not require information about absolute microbial load.

M icrobial abundance analysis is a necessary step to reveal taxonomic changes underlying a phenotype shift. For evaluations of microbial changes, however, studies that rely on relative abundances can be misleading. James Morton from the Knight group at the University of California, San Diego, notes that "to know if organisms A and B are present at $80 \%$ and $20 \%$ is one thing, but whether these numbers come from 100 or 100 million cells makes a huge difference for the host."

Yet, it is not straightforward to evaluate absolute microbial changes across samples because the generated sequencing data are compositional and inform abundance in a relative manner. Often, researchers interpret microbial changes through differential abundance analysis with known absolute bacterial loads. It is known that the quantification of microbial load can be costly and time-consuming. Moreover, it becomes a problem when one is comparing the new results with legacy data that do not include absolute abundance data.

Knight, together with his colleagues from the Zengler group, proposed a differential ranking system to measure how populations change in contrast to each other. They used the concept of 'reference frames' for inferring compositional changes of microbial populations. Instead of directly comparing relative abundance, which is likely to be biased, they used the coefficients from multinomial regression to determine relative differentials, that is, rankings identifying microorganisms that are changing the most relative to each other.
They have applied differential ranking to quantify changes of microorganisms in different types of environments. Knight comments that, "for example, it is now possible to determine what number of cells of a given microbial species will result in an immune response by the host, or to quantify turnover rates of microbes in environments ranging from the human gut to soil."

Lei Tang

Published online: 30 July 2019

https://doi.org/10.1038/s41592-019-0527-3

Research papers

Morton, J. T. et al. Establishing microbial composition measurement standards with reference frames. Nat. Commun. 10, 2719 (2019).

\section{Changing the way you see life}

Ultra Precise Motion Control - D.C. Servo motors down to $20 \mathrm{~nm}$, piezos down to $1 \mathrm{~nm}$, and low drift XYZ stages.

Microscopy - Automation, modular microscopes, autofocus complete light sheet systems, and components.

OEM - Custom designed systems to user specifications.

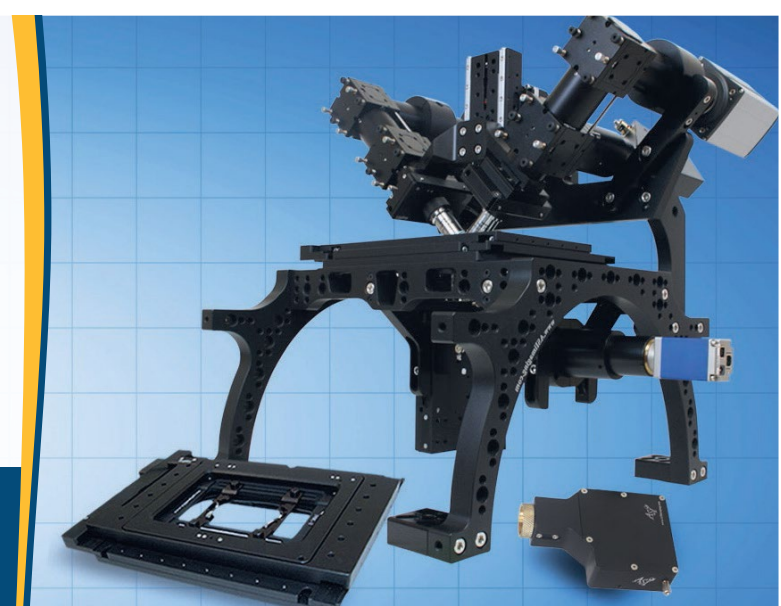

